PJOLKS

José Florencio F. Lapeña, Jr., MA, MD

Department of Otorhinolaryngology College of Medicine, University of the Philippines Manila, Philippines

Department of Otorhinolaryngology Head and Neck Surgery

East Avenue Medical Center, Diliman

Quezon City, Philippines

\section{Peer Review and the PJOHNS: Principles, Problems and Promise}

The Philippine Journal of Otolaryngology Head and Neck Surgery (PJOHNS) is the official refereed journal of the Philippine Society of Otolaryngology Head and Neck Surgery (PSOHNS). What does it mean for our journal to be refereed or peer reviewed? According to the World Association of Medical Editors (WAME), ${ }^{1}$

"A peer-reviewed biomedical journal is one that regularly obtains advice on individual manuscripts from reviewers who are not part of the journal's editorial staff. Peer review is intended to improve the accuracy, clarity and completeness of published manuscripts and to help editors decide which manuscripts to publish. Peer review does not guarantee manuscript quality and does not reliably detect scientific misconduct."

Who are peer reviewers? Integral to the whole system, they are experts in their chosen field who are expected to provide an unbiased opinion on the quality, timeliness and relevance of a submitted manuscript. ${ }^{2}$ They are responsible to the editor and journal, their specialty and/or subspecialty, study participants and/or subjects, and authors, to "make sure rubbish does not get published."2

As editors, we understand that peer review (also called refereeing) is not a perfect antidote to poor science and we need to carefully evaluate manuscripts themselves for quality and validity. ${ }^{3}$ Prior to review, we carefully review submissions for suitability to our journal and ensure that all important elements of the manuscript are included, in accordance with our instructions to authors. ${ }^{4}$ Manuscripts are subjected to a double blinded external peer-review process, guided by the "Responsibilities and Rights of Peer Reviewers" contained in the Editorial Policy Statements approved by the Council of Science Editors Board of Directors. ${ }^{5}$ For participants in the PSOHNS research contests, this review process is facilitated by pre-judging of anonymized manuscripts by blinded judge-reviewers. Manuscripts are further reviewed by editors and other experts in the field and may be proofread, content- and form- edited and returned for revision. The revision process is often tedious, particularly when authors fail to adequately address the concerns, comments and corrections of editors and reviewers (or referees). In this regard, authors have much to learn from research protocol and medical writing workshops. It is also in the best interests of editors and their journals to improve peer review and ways to do so have been identified by systematic reviews. ${ }^{6,7}$ 
Double-blind review (blinding both author and reviewer to each other's identity and anonymizing manuscripts before review) supposedly reduces the likelihood of bias for or against authors based on name, affiliation or country of origin and is perceived as more fair. ${ }^{3,8}$ Unless they are able to guess the identity of authors, reviewers only discover such identities if the manuscript is finally accepted and published. ${ }^{7,9}$ On the other hand, lack of transparency may be considered a limitation of double-blind review although the cultural-appropriateness of transparency may be argued in collective cultural contexts such as ours. To minimize bias and manage and assure the quality of the peer review process, we try to select peer reviewers who possess the appropriate expertise needed to review a manuscript thoroughly and identify and exclude peer reviewers with potential conflicts of interest. ${ }^{10}$

In cooperation with the PSOHNS, we conduct 1-day introduction to basic medical writing workshops, and 2-day advanced workshops for peer reviewers every year as well as mini-workshops during our annual convention. Unfortunately, it seems that those who would benefit most from our courses are not the ones who participate in them. Very few consultants have attended either workshop, evinced by the quality of co-authorship of their own papers, or their reviews of other papers. There are many instances where senior colleagues perpetuate inappropriate research and writing practices, contradicting what would have been the correct work of their junior co-authors (the latter merely applying what they learned from our workshops). Worse, some of these consultants insist on their way (as research supervisors, co-authors or reviewers), undoing our corrections and misguiding residents in the process. Things would be different if they opened themselves to acquiring the knowledge, skills, and attitudes of good referees, and contribute to the ongoing history of scholarship in our field. Meanwhile, regardless of the recommendations they make, the ultimate decision and responsibility is the editor's.

We aim to publish original work of value to the intellectual community in the best possible form and to the highest possible standards and expect similar standards from our reviewers and authors. Our journal follows the "Recommendations for the Conduct, Reporting, Editing and Publication of Scholarly Work in Medical Journals" of the International Committee of Medical Journal Editors (ICMJE), and is proudly listed as such." Honesty, originality and fair dealing on the part of authors, and fairness, objectivity and confidentiality on the part of editors and reviewers are among the critical values that enable us to achieve our aim. To this end, we also endorse and uphold the Ethical Guidelines for Peer Reviewers established by the Committee on Publication Ethics (COPE). ${ }^{12}$

We hope that our efforts are well worth the hardships and heartaches we endure with each manuscript we process. Guided by our principles, we painstakingly search for solutions to current problems as the promise of a better tomorrow beckons. We invite you to partner with us as peer reviewers and participate in our future.

\section{REFERENCES}

1. World Association of Medical Editors. Definition of a Peer-Reviewed Journal. Oct 19, 2007. [Cited 26 May 2018]. Available from: http://www.wame.org/policy-statements\#Definition\%20 PR.

2. Peh WC, Ng KH. Role of the Manuscript Reviewer. Singapore Med J 2009 Oct; 50(10): 931-933. PMID:19907880.

3. Lapeña JF, Winker M. Peer Review, Manuscript Decisions, and Author Correspondence. WAME eLearning Program. World Association of Medical Editors 2018. (Forthcoming).

4. Philippine Journal of Otolaryngology Head and Neck Surgery. Instructions to Authors. [Cited 26 May 2018]. Available from: https://journal.pso-hns.org/instructions-to-authors/.

5. Council of Science Editors. Responsibilities and Rights of Peer Reviewers. CSE Editorial Policy Statement. Science Editor 2002 Nov-Dec;25(6):187. [Cited 26 May 2018] Available from: https:// www.councilscienceeditors.org/wp-content/uploads/v25n6p187.pdf.

6. Jefferson T, Rudin M, Brodney Folse S, Davidoff F. Editorial peer review for improving the quality of reports of biomedical studies. Cochrane Database of Systematic Reviews 2007, Issue 2. Art. No.: MR000016. DOI: 10.1002/14651858.MR000016.pub3.

7. Bruce R, Chauvin A, Trinquart L, Ravaud P, Boutron I. Impact of interventions to improve the quality of peer review of biomedical journals: a systematic review and meta-analysis. BMC Med.
2016 Jun;14(1):85. DOI: 10.1186/s12916-016-0631-5 PMID: 27287500 PMCID: PMC4902984.

8. Okike K, Hug KT, Kocher MS, Leopold SS. Single-blind vs Double-blind Peer Review in the Setting of Author Prestige. JAMA. 2016 Sep;316(12):1315-1316. DOI:10.1001/jama.2016.11014 PMID:27673310.

9. Justice AC, Cho MK, Winker MA, Berlin JA, Rennie D, and the PEER Investigators. Does Masking Author Identity Improve Peer Review Quality? A Randomized Controlled Trial. JAMA. 1998 Jul;280(3):240-242. DOI:10.1001/jama.280.3.240 PMID:9676668.

10. Moher D, Galipeau J, Alam S, Barbour V, Bartolomeos K, Baskin P, et al. Core competencies for scientific editors of biomedical journals: consensus statement. BMCMed. 2017 Sep 11;15(1):167. DOI: 10.1186/s12916-017-0927-0. PMID:28893269 PMCID: PMC5592713 [Cited 26 May 2018] Available from: https://bmcmedicine.biomedcentral.com/articles/10.1186/s12916-017-0927-0.

11. International Committee of Medical Journal Editors (ICMJE). Recommendations for the Conduct Reporting, Editing, and Publication of Scholarly Work in Medical Journals. [Cited 26 May 2018] Available from: http://www.icmje.org/icmje-recommendations.pdf.

12. COPE Council. Ethical Guidelines for Peer Reviewers. September 2017. [Cited 26 May 2018] Available from: https://publicationethics.org/files/Ethical_Guidelines_For_Peer_Reviewers_2. pdf. 